Supporting Information

\title{
Penetration Cascade of Size Switchable Nanosystem in Desmoplastic Stroma for Improved Pancreatic Cancer Therapy
}

Xiaozheng Zhao ${ }^{1,2, \#}$, Xiao Yang ${ }^{1, \#}$, Xudong Wang ${ }^{1, \#}, X_{i a o} Z_{h a o^{1}}$, Yinlong Zhang ${ }^{1}$, Shaoli Liu ${ }^{1,2}$, Gregory J. Anderson ${ }^{3}$, Seong-jin Kim ${ }^{4,5 *}$, Yiye Li $^{1,2, *}$, Guangjun Nie ${ }^{1,2,6 *}$

${ }^{1}$ CAS Key Laboratory for Biomedical Effects of Nanomaterials and Nanosafety, CAS Center for Excellence in Nanoscience, National Center for Nanoscience and Technology, Beijing 100190, P.R.China

${ }^{2}$ Center of Materials Science and Optoelectronics Engineering, University of Chinese Academy of Sciences, Beijing 100049, P.R.China

${ }^{3}$ QIMR Berghofer Medical Research Institute, Royal Brisbane Hospital, Brisbane, Queensland 4029, Australia

${ }^{4}$ GILO Institute, GILO Foundation, Seoul 06668, Republic of Korea

${ }^{5}$ Medpacto Inc., Myeongdal-ro, Seocho-gu, Seoul 06668, Republic of Korea

${ }^{6}$ GBA Research Innovation Institute for Nanotechnology, Guangdong 510700, China

Corresponding authors:

*E-mail: liyy@ nanoctr.cn (Yiye Li)

*E-mail: jasonsjkim@gilo.or.kr (Seong-jin Kim)

*E-mail: niegj@nanoctr.cn (Guangjun Nie)

\#These authors contributed equally to this work. 
Table S1. VAC loading efficiency

\begin{tabular}{ccc}
\hline $\begin{array}{c}\text { Liposomes: VAC } \\
\text { (W/W) }\end{array}$ & Loading efficiency & $\begin{array}{c}\text { Encapsulating } \\
\text { Efficiency }\end{array}$ \\
\hline $50: 1$ & $1.2 \%$ & $60.0 \%$ \\
$50: 2$ & $2.0 \%$ & $50.0 \%$ \\
$50: 5$ & $4.6 \%$ & $46.0 \%$ \\
$50: 10$ & $5.0 \%$ & $25.0 \%$ \\
$50: 20$ & $5.2 \%$ & $13.0 \%$ \\
\hline
\end{tabular}

Table S2. TAX loading efficiency

\begin{tabular}{ccc}
\hline $\begin{array}{c}\text { Nanosphere:TAX } \\
\text { (W/W) }\end{array}$ & Loading efficiency & $\begin{array}{c}\text { Encapsulating } \\
\text { Efficiency }\end{array}$ \\
\hline $20: 0.1$ & $0.35 \%$ & $70.0 \%$ \\
$20: 0.2$ & $0.50 \%$ & $50.0 \%$ \\
$20: 0.5$ & $1.35 \%$ & $54.0 \%$ \\
$20: 1$ & $2.00 \%$ & $40.0 \%$ \\
\hline
\end{tabular}


A

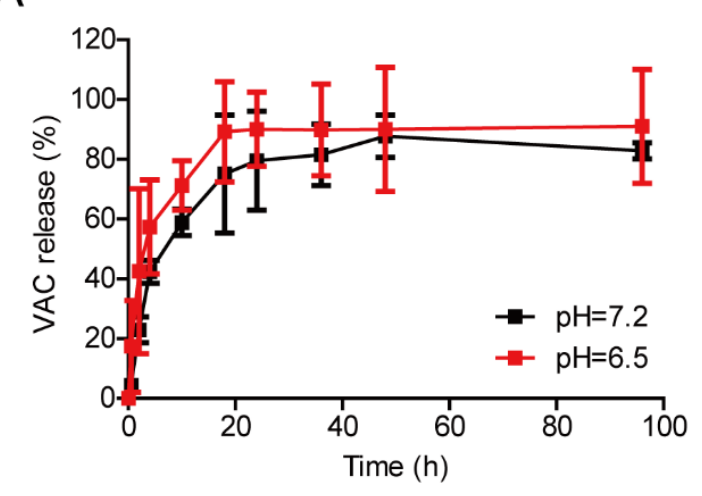

C

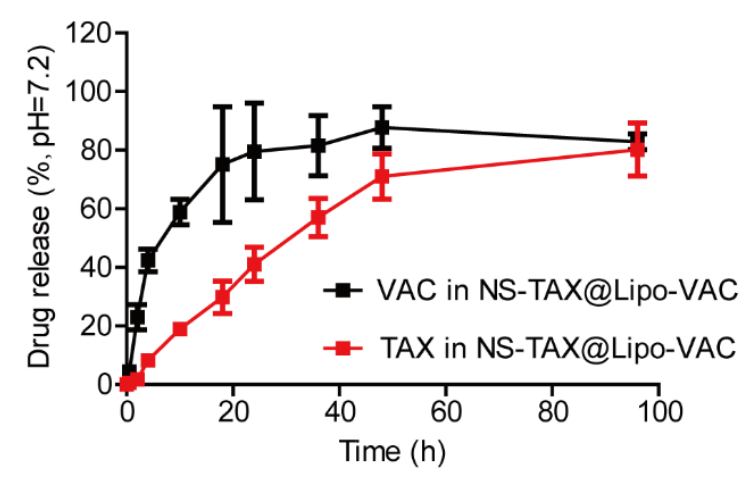

B

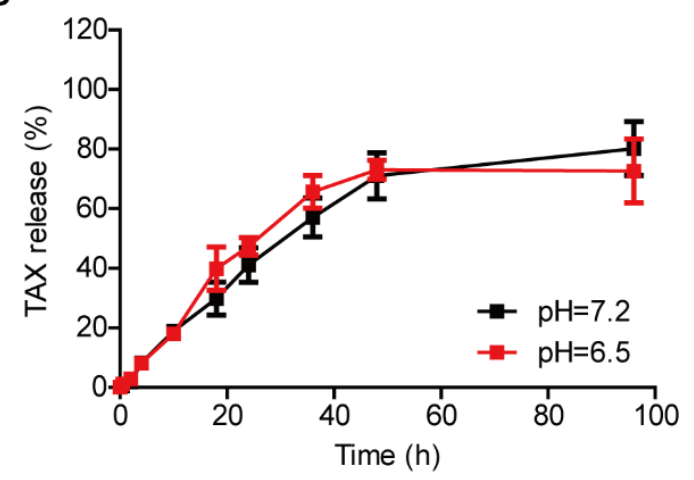

D

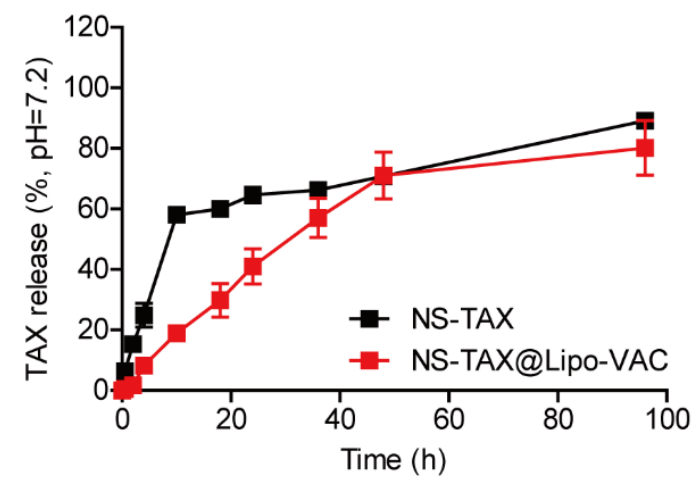

Figure S1. Drug release of NS-TAX@Lipo-VAC. A. The accumulated VAC release profiles of NS-TAX@Lipo-VAC in PBS. B. The accumulated TAX release profiles of NS-TAX@LipoVAC in PBS. C. The accumulated drug release profiles of NS-TAX@Lipo-VAC in PBS $(\mathrm{pH}=7.2)$. D. The accumulated TAX release profiles of NS-TAX@Lipo-VAC and NS-TAX in PBS ( $\mathrm{pH}=7.2)$. 


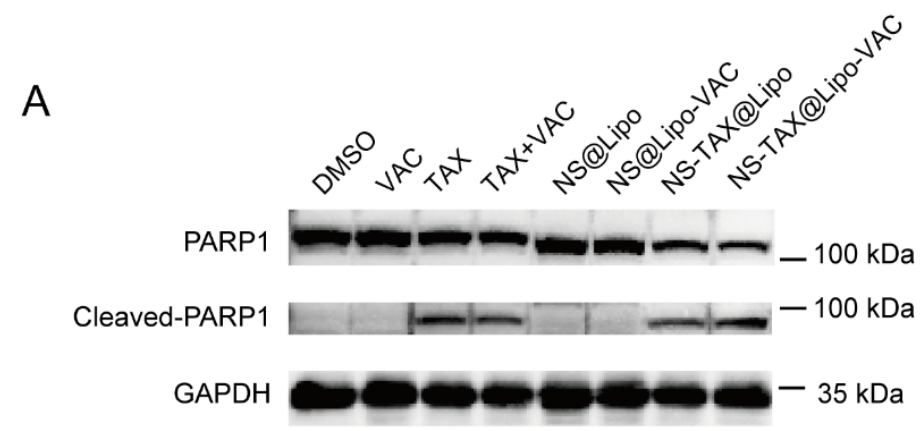

B
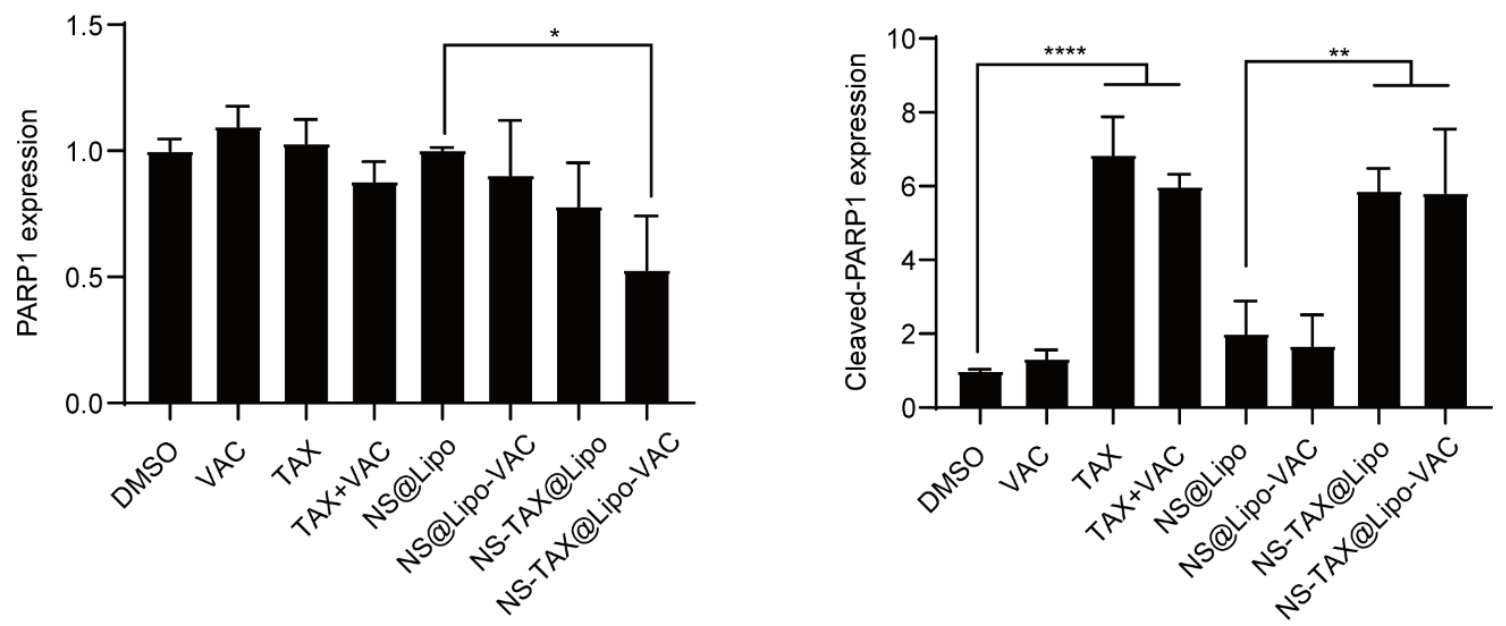

Figure S2. Western blot analysis of cleaved-PARP1 in Panc-1 cells after different treatments for 48 hours (A) and quantitative analysis of the normalized PARP1 and cleaved-PARP1 levels in Panc-1 upon different treatments $(\mathbf{B})$. The data are shown as the mean \pm s.d. $(\mathrm{n}=3) * p<0.05$, ${ }^{* *} p<0.01, * * * * p<0.0001$ (One-way ANOVA analysis). 


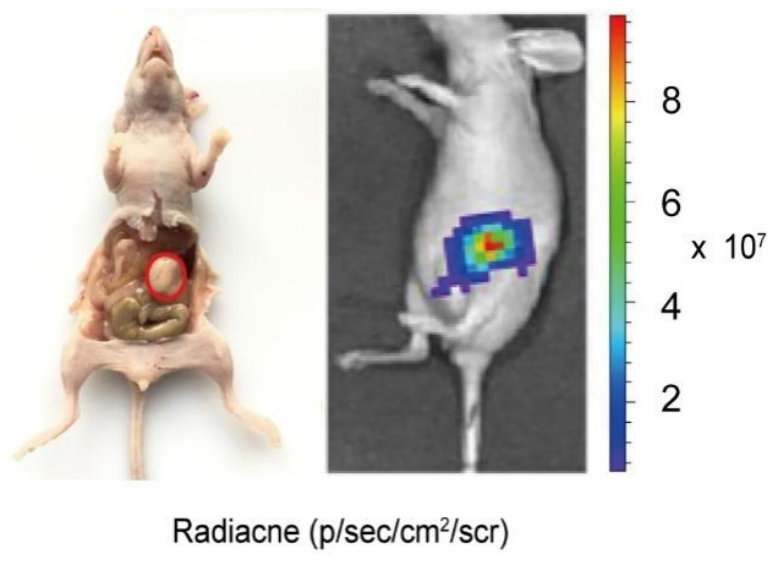

Figure S3. Image (left) and bioluminescent imaging (right) of established pancreatic orthotopic tumor models in situ. The tumor region was circled in red color on the left image. 

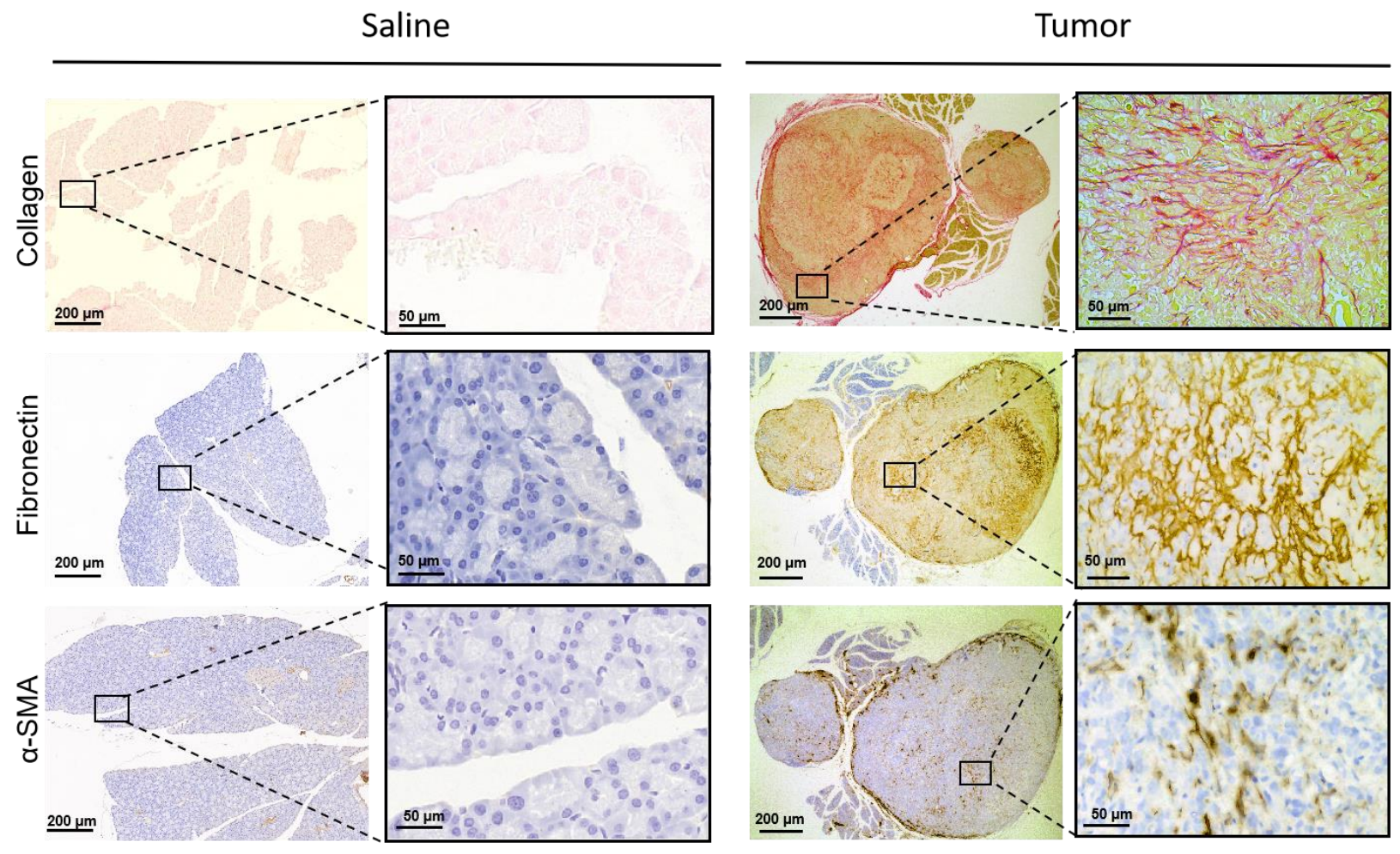

Figure S4. Characterization of microenvironment of orthotopic pancreatic tumor model. Representative total collagen was chemically stained by Sirius Red and immunohistochemistry staining of fibronectin and $\alpha$-SMA indicated the ECM deposition and presence of PSC. 


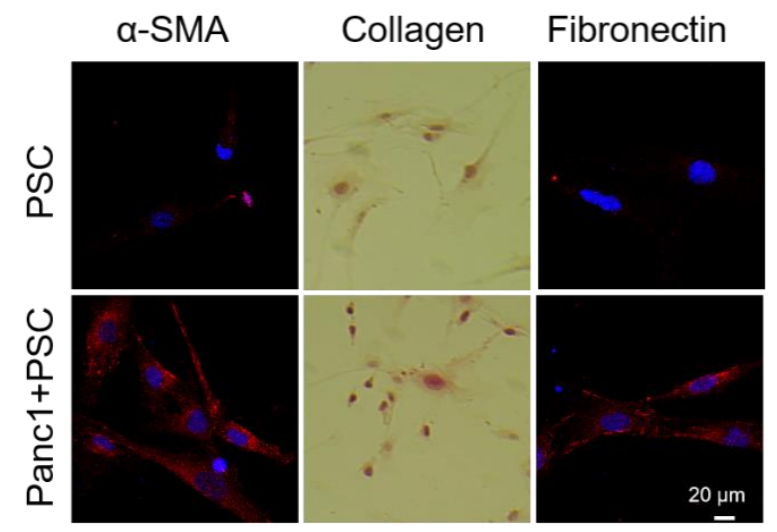

Figure S5. Immunofluorescence staining of $\alpha$-SMA, collagen and fibronectin in co-culture of murine PSCs and Panc-1. The deposited collagen is chemically stained by Sirius Red. 

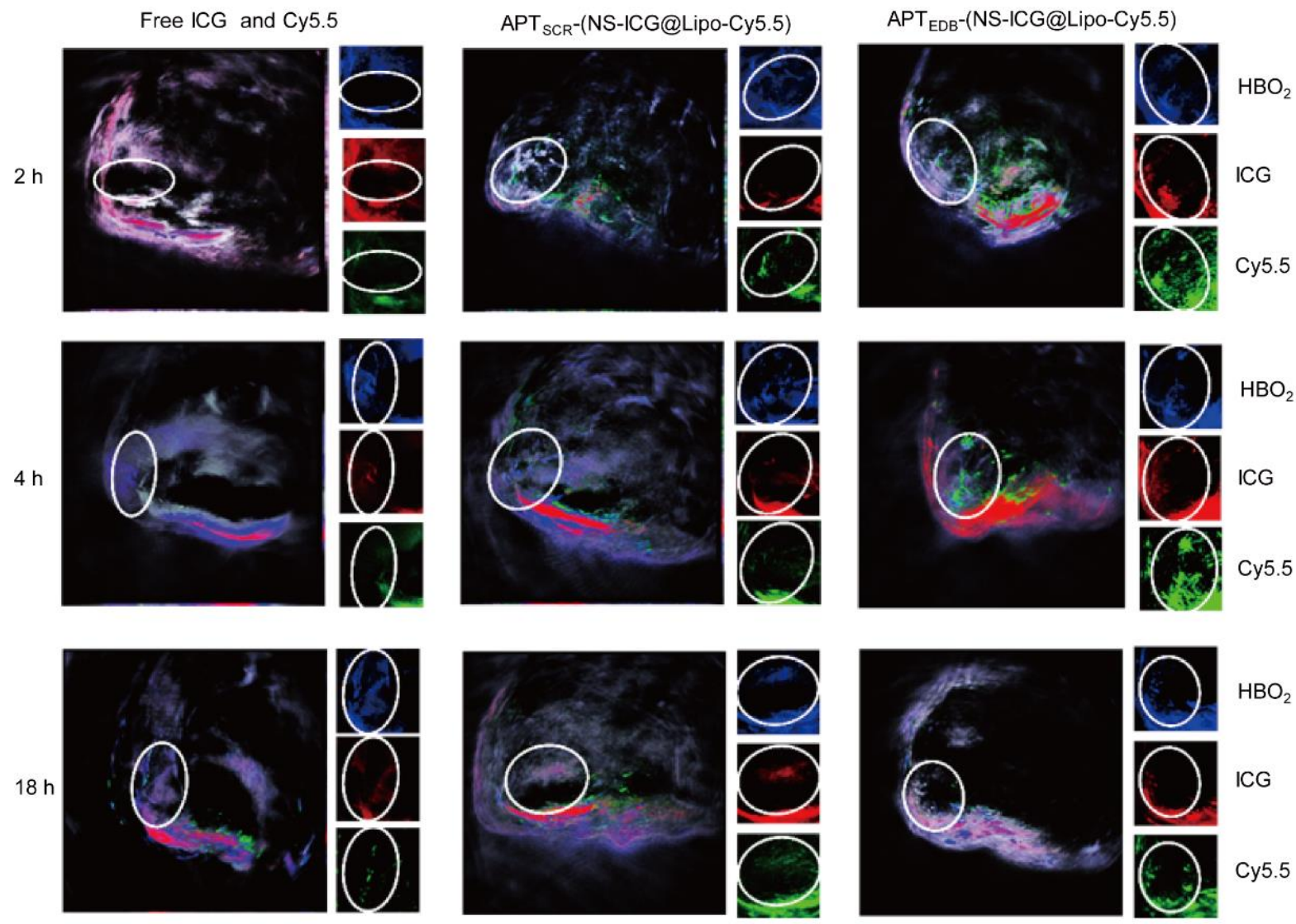

$\mathrm{HBO}_{2}$

ICG

Cy5.5

Figure S6. Targeting efficiency of $\mathrm{APT}_{\mathrm{EDB}}$ in vivo. In vivo photoacoustic images of the tumor areas (white ovals) after intravenous injection of indocyanine green (ICG)- and Cy5.5-labeled nanoparticles for different time. 
A

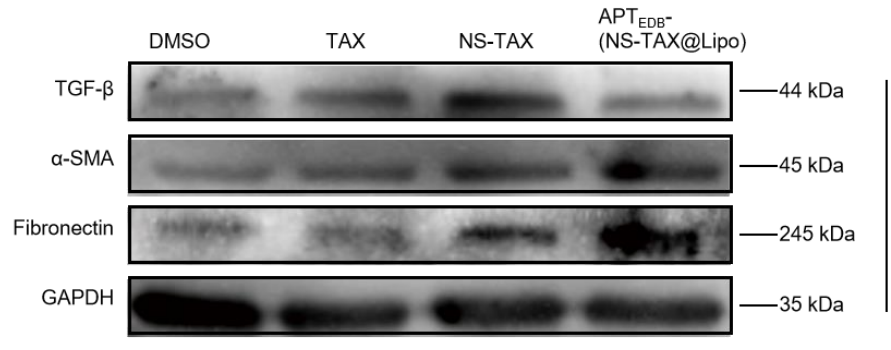

Cellular proteins

Fibronectin

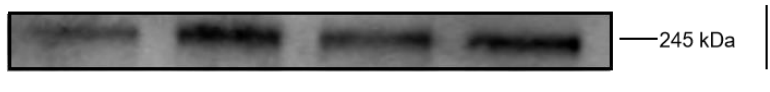

Secreted proteins
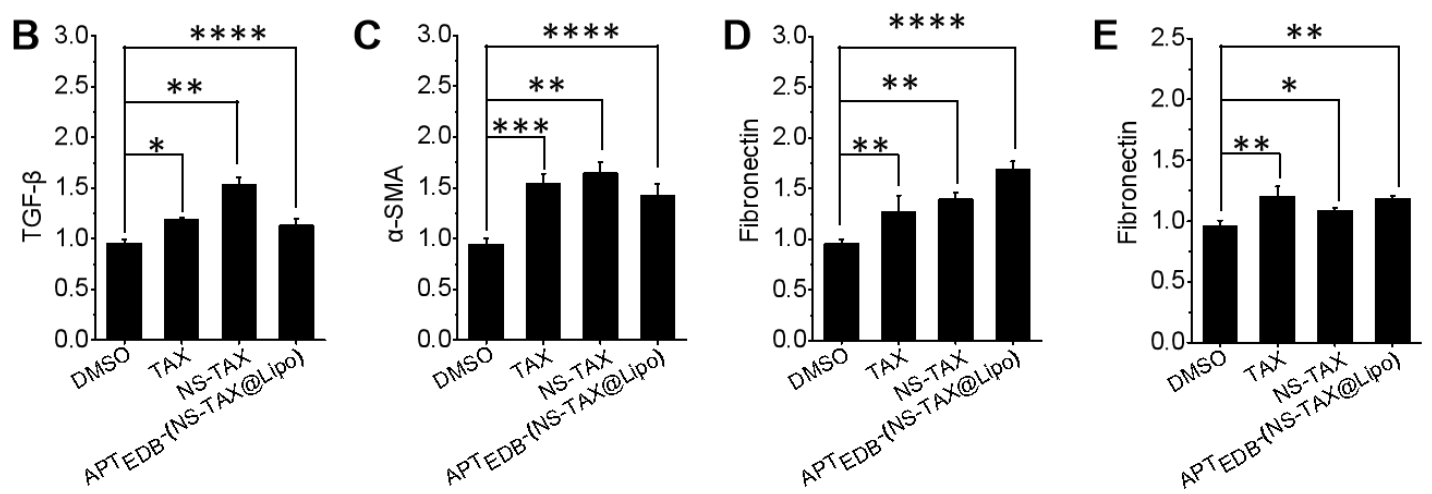

Figure S7. Western blot analysis of TGF- $\beta, \alpha$-SMA and fibronectin expression of PSCs after treatment with TAX, NS-TAX or APTEDB-(NS-TAX@Lipo) for 48 hours (A). Quantitative analysis of the normalized cellular expression of TGF- $\beta$ (B), $\alpha$-SMA (C), fibronectin (D), and secreted fibronectin (E) in PSCs upon different treatment. The data are shown as the mean \pm s.d. $(\mathrm{n}=3) * p<0.05, * * p<0.01, * * * p<0.001, * * * * p<0.0001$ compared to control group. 


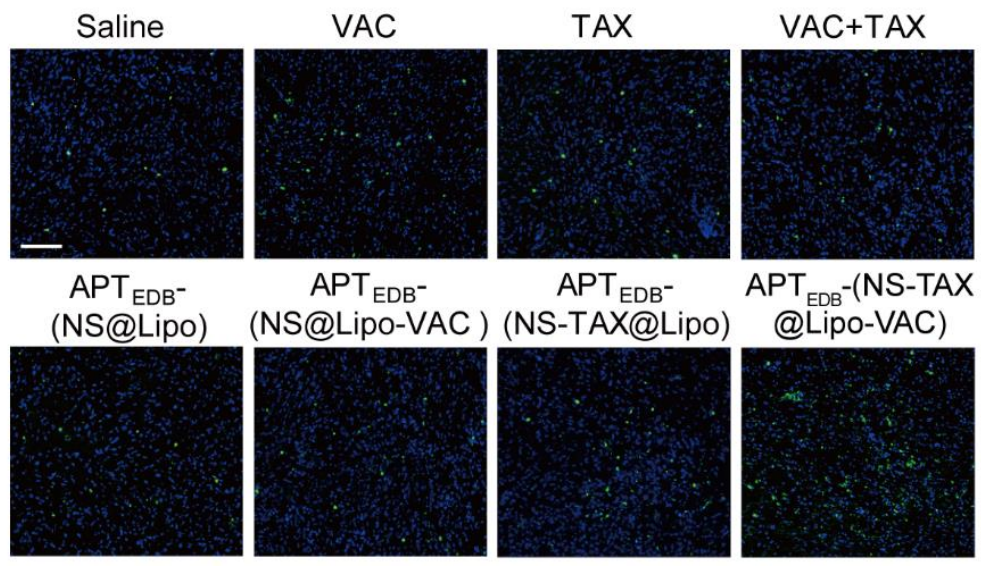

Figure S8. TUNEL analysis of tumor section. Scale bar, $100 \mu \mathrm{m}$. 


\section{A}

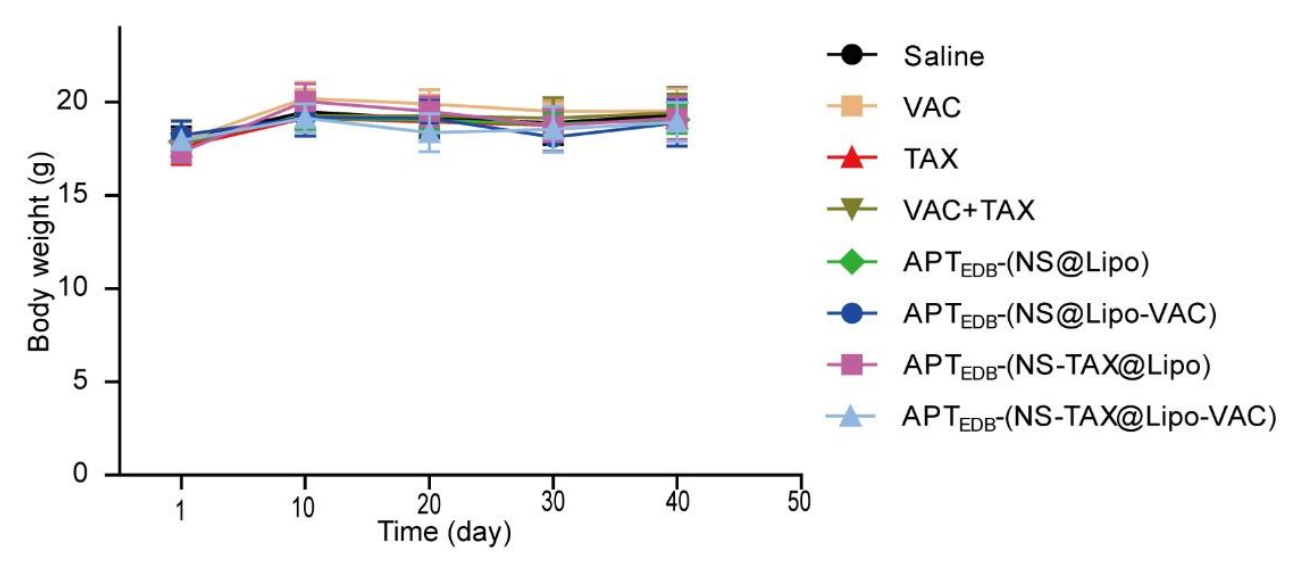

B

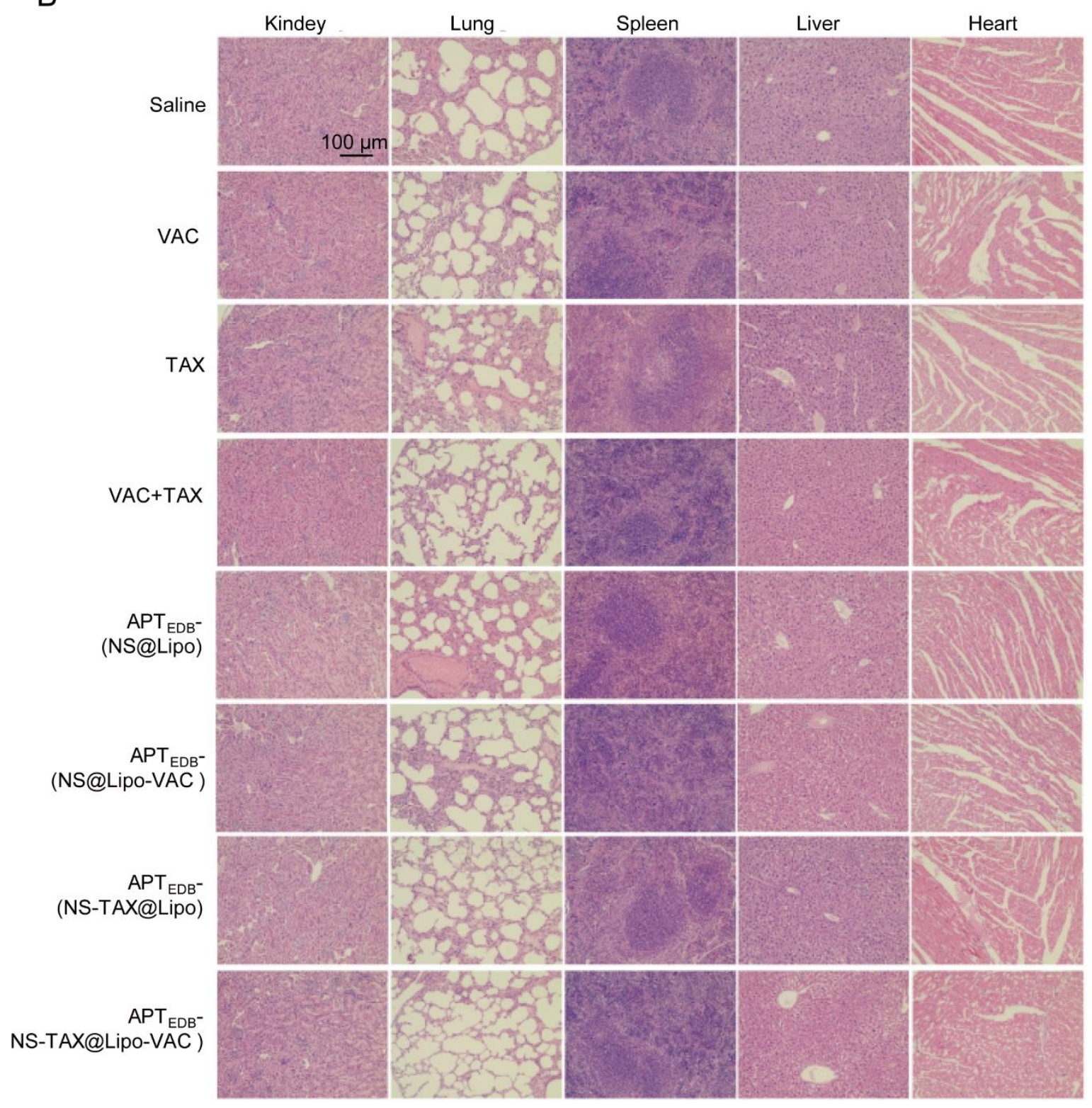


Figure S9. Safety profile of different treatments. A. Body weight of tumor-bearing mice weighed during the treatments. B. Representative H\&E staining of the major organs of tumorbearing mice after different treatments. The kidney, lung, spleen, liver, and heart of tumorbearing mice showed no visible pathological changes. 